\title{
Pathologic Stage IC Gastroesophageal Junction Adenocarcinoma AJCC v8
}

National Cancer Institute

\section{Source}

National Cancer Institute. Pathologic Stage IC Gastroesophageal Junction

Adenocarcinoma A/CC v8. NCI Thesaurus. Code C133568.

Stage IC includes: (T1, N0, M0, G3); (T2, N0, M0, G1-2). T1: Tumor invades the lamina propria, muscularis mucosae, or submucosa. T2: Tumor invades the muscularis propria. N0: No regional lymph node metastasis. M0: No distant metastasis. G1: Welldifferentiated. G2: Moderately differentiated. G3: Poorly differentiated, undifferentiated. (AJCC 8th ed.) 